\title{
Axial gravitational waves in FLRW cosmology and memory effects
}

\author{
Wojciech Kulczycki and Edward Malec \\ Instytut Fizyki Mariana Smoluchowskiego, Uniwersytet Jagielloński, Eojasiewicza 11, 30-348 Kraków, Poland
}

\begin{abstract}
We show initial data for gravitational axial waves, that are twice differentiable but which are not $C^{2}$. They generate wave pulses that interact with matter in the radiation cosmological era. This forces the radiation matter to rotate. This rotation is permanent - it persists after the passage of the gravitational pulse. The observed inhomogeneities of the cosmic microwave background radiation put a bound onto discontinuities of superhorizon metric perturbations. We explicitly show that a class of smooth initial metrics that are at least $C^{2}$ gives rise to gravitational wave pulses that do not interact with the background during the radiation epoch.
\end{abstract}

\section{INTRODUCTION}

Memory effects are known in electrodynamics of infrared fields; if a test body moves through a pulse of zeroenergy photons, then its momentum is conserved, but its trajectory will be shifted [1] (see commentaries on this effect and later literature in [2]). In general relativity, Zeldovitch and Polnarev [3] have shown, within linearized gravity, that a test body is permanently displaced by a special type of passing gravitational waves. The characteristic feature of these effects is the permanent distortion of the metric after the passage of the (curvature sandwichtype) wave pulses [4 7]. This means that these wave pulses are dominated by zero-energy gravitons (see [8] for a discussion). Usually these are weak signals; for the detection of GW150914 [9] the memory strain is 20 times weaker than the oscillatory strain [10, but under certain circumstances the memory strain can be much larger than the oscillatory one [11].

In this paper we discuss the propagation of explicit gravitational (perturbing metric sandwich-type) waves within radiative Friedmann-Lemaitre-Robertson-Walker flat space-times. Such gravitational waves do not leave permanent traces on the relative distance of two test bodies - it is the same before and after the passage of the pulse. Their Fourier transforms are not dominated by zero-energy frequencies and the waves are not dominated by zeroenergy gravitons. Nevertheless, we show that these waves leave permanent tracks in the radiation background, if their initial profiles are somewhat deficient in smoothness - the metric perturbations are twice differentiable, but their second derivatives are not continuous. In such cases passing wave pulses force the radiation fluid to rotate.

Let us remark that there is no necessity to assume high orders of differentiability of solutions of wave equations, even if these equations are nonlinear. Gravitational wave profiles do not have to be smooth; they can be even distribution valued. Let us recall the explicit construction of $\delta$-like gravitational waves by Penrose [12] (see also a discussion by Luk and Rodnianski [13]).

We use the Regge-Wheeler formalism. Regge and Wheeler imposed a gauge condition that allows for the ex- traction of the two gauge-independent linearized modes ("axial" and "polar") of gravitational waves [14. Their original analysis (corrected later by Zerilli [15]) dealt with gravitational waves in the Schwarzschild space-time, but their approach appeared convenient in the context of cosmology [16 22]. There exists an independent (and equivalent) line of research, with the use of gauge-invariant quantities [23 25]. Clarkson et al. discussed cosmological perturbations in the wider context of LemaitreTolman cosmologies 25; their equations, restricted to the Friedmann-Lemaitre-Robertson-Walker metrics deformed by axial modes, coincide with those of [16, 21].

We assume gravitational units $c=8 \pi G=1$.

\section{EQUATIONS}

We shall use standard coordinates $(\eta, r, \theta, \phi)$, but with the conformal time coordinate:

$$
d s^{2}=a^{2}(\eta)\left(-d \eta^{2}+d r^{2}+r^{2} d \theta^{2}+r^{2} \sin ^{2} \theta d \phi^{2}\right) .
$$

We restrict our attention to the flat $k=0$ universe.

We shall use below the "conformal" Hubble constant,

$$
H(\eta):=\frac{\partial_{\eta} a}{a},
$$

which is related to the "ordinary" Hubble constant $\underline{H}$ by $\underline{H}=\frac{H}{a}$.

Below we put for notational simplicity $Y=Y(\theta):=$ $Y_{l 0}(\theta)$ and $Y^{\prime}=\partial_{\theta} Y$, where $Y_{l m}$ are the spherical harmonics.

The axially perturbed components of the metric in the Regge-Wheeler gauge read

$$
g_{\mu \nu}=a^{2}(\eta) \eta_{\mu \nu}^{(0)}+h_{\mu \nu}
$$

where $\eta_{\mu \nu}^{(0)}$ is the Minkowski metric and the only nonzero components of $h_{\mu \nu}$ are

$$
h_{0 \phi}=h_{0} \sin \theta Y^{\prime} \quad h_{r \phi}=h_{1} \sin \theta Y^{\prime} .
$$


Here $h_{0}=h_{0}(\eta, r), h_{1}=h_{1}(\eta, r)$. The material field is described by the stress-energy tensor

$$
T_{\mu \nu}=\left(\rho_{0}+p_{0}\right) u_{\mu} u_{\nu}+p_{0} g_{\mu \nu}-\Lambda g_{\mu \nu} .
$$

It has been already shown in 21 that the mass density $\rho_{0}$ and the pressure $p_{0}$ cannot be disturbed by the axial modes. Finally, one should allow for the possibility that matter is not necessarily comoving with the unperturbed cosmological expansion. It appears that axial modes of the gravitational waves can affect only two components of the 4 -velocity of matter [21. We have, up to terms linear in perturbations,

$$
\begin{aligned}
u_{0} & =-a(\eta), \\
u_{\phi} & =\sin \theta \cdot u(\eta, r) Y^{\prime}
\end{aligned}
$$

This ensures that $u_{\mu} u^{\mu}=-1$ up to linear terms.

The (background) isotropic and homogeneous solution of Friedmann equations satisfies the following relations:

$$
\rho_{0}=\frac{3}{a^{2}} H^{2}-\Lambda
$$

and

$$
p_{0}=\Lambda-\frac{1}{a^{2}} H^{2}-\frac{2}{a^{2}} \frac{d H}{d \eta} .
$$

From these two equations one arrives at

$$
\frac{a^{2}}{2}\left(\frac{1}{3} \rho_{0}-p_{0}+\frac{4}{3} \Lambda\right)=H^{2}+\frac{d H}{d \eta} .
$$

We also need (see Sec. IV) the continuity equation

$$
\frac{d \rho_{0}}{d \eta}=-3 H\left(p_{0}+\rho_{0}\right)
$$

Linearized Einstein equations corresponding to the metric 3 read

$$
\partial_{r} h_{1}=\partial_{\eta} h_{0}
$$

$$
\begin{aligned}
& \partial_{r} \partial_{\eta} h_{1}-\partial_{r}^{2} h_{0}-2 H \partial_{r} h_{1}+\frac{2}{r} \partial_{\eta} h_{1}-\frac{4 H}{r} h_{1}+ \\
& +\frac{l(l+1)}{r^{2}} h_{0}=-2 a^{3}\left(\rho_{0}+p_{0}\right) u
\end{aligned}
$$

$$
\begin{aligned}
& \partial_{\eta}^{2} h_{1}-\partial_{r} \partial_{\eta} h_{0}-2 H \partial_{\eta} h_{1}+\frac{2}{r} \partial_{\eta} h_{0}-2 \frac{d H}{d \eta} h_{1}+ \\
& \frac{l(l+1)-2}{r^{2}} h_{1}=0 .
\end{aligned}
$$
by

Inserting (11) into (13), defining a new quantity $Q(\eta, r)$

$$
h_{1}(\eta, r)=r a(\eta) Q(\eta, r),
$$

and using 9 , one gets 21

$\partial_{\eta}^{2} Q-\partial_{r}^{2} Q+\frac{l(l+1)}{r^{2}} Q-\frac{1}{2} a^{2}\left(\frac{1}{3} \rho_{0}-p_{0}+\frac{4}{3} \Lambda\right) Q=0$.

The master equation (15) can be solved independently of the remaining equations - it describes one of the two independent gravitational modes.

From Eq. (11) one gets $h_{0}$ :

$$
h_{0}(\eta, r)=A(r)+\int_{\eta_{0}}^{\eta} h_{1}^{\prime}(\tau, r) d \tau
$$

where $\eta_{0}$ characterizes the initial hypersurface. The function $A(r)$ is arbitrary, but if $h_{0}(\eta, r)$ vanishes at the initial hypersurface, then $A(r)=0$. We assume that $h_{0}\left(\eta_{0}, r\right)=0$.

\section{AXIAL GRAVITATIONAL WAVES AND COSMOLOGICAL ROTATION}

We shall assume the radiation fluid with the equation of state $p_{0}=\frac{\rho_{0}}{3}$ and $\Lambda=0$. Then the conformal factor $a(\eta)=b \eta$, with $b$ being a constant of the dimension (length) $)^{-1}$, and the energy density $\rho_{0} \propto \eta^{-4}$. We restrict our attention to quadrupole $(l=2)$ modes. During the radiation epoch, in the absence of the cosmological constant, the quadrupole master equation reads

$$
\partial_{\eta}^{2} Q-\partial_{r}^{2} Q+\frac{6}{r^{2}} Q=0
$$

Its general solution has the form (see, for instance, [17])

$$
Q(r, \eta)=r^{2} \partial_{r} \frac{1}{r} \partial_{r}\left(\frac{g+h}{r}\right)
$$

where functions $g$ and $h$ depend on the combinations $r-\eta$ or $r+\eta$, respectively.

The outgoing gravitational wave is represented by

$$
Q(r, \eta)=r^{2} \partial_{r} \frac{1}{r} \partial_{r}\left(\frac{g(r-\eta)}{r}\right)
$$




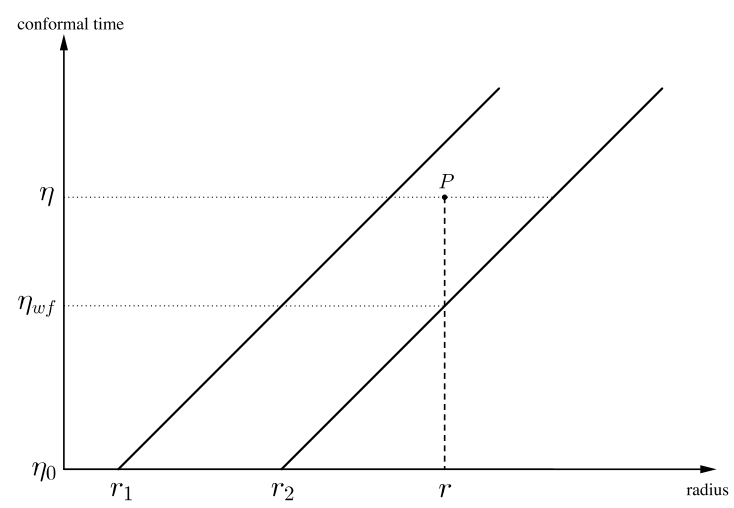

FIG. 1. Space-time diagram showing the propagation of the considered gravitational pulse $\left(\eta_{w f}=r-r_{2}+\eta_{0}\right)$. The dashed line shows the integration contour for the integral in 16 .

Assume compact initial data at $\eta=\eta_{0}$, with the initial support of $g$ contained within the annulus $r_{1} \leq r \leq r_{2}$. Let them be at least four times differentiable everywhere except for isolated points. For the sake of simplicity we assume that there is only one discontinuity at the wave front. This means that the space-time metric is at least twice differentiable everywhere with the exception of the wave front. The wave front of this initial pulse is represented by the sphere $r=r_{2}+\eta-\eta_{0}$. A straightforward calculation [see Eq. 12]] shows that if this wave passes through a point $P$ of coordinates $(r, \eta)$, with $r>r_{2}$ (see Fig. 1), then $P$ acquires azimuthal velocity; the quantity

$$
\begin{aligned}
u(r, \eta) \rho_{0} a^{3}= & b \frac{9 g\left(r_{2}-\eta_{0}\right)}{8 r^{2}}+\left.b \frac{9\left(r_{2}-\eta_{0}\right)}{8 r^{2}} \partial_{\eta} g\left(r_{2}-\eta\right)\right|_{\eta_{0}}- \\
& \left.b \frac{9\left(r-r_{2}+\eta_{0}\right)}{8 r} \partial_{\eta}^{2} g\left(r_{2}-\eta\right)\right|_{\eta_{0}}- \\
& \left.b \frac{3 r}{8} \partial_{\eta}^{3} g\left(r_{2}-\eta\right)\right|_{\eta_{0}}+ \\
& \left.b \frac{3 r}{8}\left(r-r_{2}+\eta_{0}\right) \partial_{\eta}^{4} g\left(r_{2}-\eta\right)\right|_{\eta_{0}} .
\end{aligned}
$$

becomes nonzero. We stress that this effect depends only on characteristics of the discontinuity of $g$, which is located at the wave front of the pulse. For smooth initial profiles, with $g \in C^{k}(k \geq 4)$, the right-hand side of (20) vanishes. Notice that the angular velocity of fluid is proportional to $u, \Omega=\frac{u^{\phi}}{u^{0}}=C \frac{u \cos \theta}{a r^{2}}$. Thus wave profiles, that are at least $C^{4}$, do not enforce cosmological rotation. Smooth pulses of axial gravitational waves travel through the cosmological radiative space-time without disturbing the radiation fluid, in agreement with the assumptions adopted in [16, 25].

We work in the linearization regime, which means that perturbed linear velocities of particles of fluid should be small compared to their Hubble flow velocities. That implies that we can allow only a mild discontinuity that the fourth derivative of $g$ is finite. Thence the first three derivatives of $g$ must vanish at the wave front. Then one finds

$$
u(r, \eta) \rho_{0} a^{3}=\left.b \frac{3 r}{8}\left(r-r_{2}+\eta_{0}\right) \partial_{\eta}^{4} g\left(r_{2}-\eta\right)\right|_{\eta_{0}},
$$

which is nonzero, if $\partial_{\eta}^{4} g$ has a discontinuity at the wave front. In such a case the passage of a wave front through a particle of fluid forces it to rotate around the symmetry axis, with the angular velocity $\Omega$. This effect is permanent - the rotation persists after the wave leaves the region.

\section{DISCUSSION}

What kind of physical processes would be responsible for discontinuities such as those discussed above? In order to gain insight, one would have to transform the RW perturbations into their standard harmonic form, with $h_{T T}$ (transversal and traceless) perturbations. In the first step one defines a new variable $\tilde{\phi}=\phi+X^{\phi}$, where $X^{\phi} \equiv \frac{Y^{\prime} \int_{\eta_{0}}^{\eta} d \tau h_{0}}{a^{2} r^{2} \sin \theta}$ (with remaining variables unchanged, $\left.X^{\eta}=X^{r}=X^{\theta}=0\right)$. The new perturbation metric terms would be found by $\tilde{h}_{\mu \nu}=h_{\mu \nu}-\nabla_{\mu} X_{\nu}-\nabla_{\nu} X_{\mu}$. The components $\tilde{h}_{0 \mu}$ would all vanish, while $\tilde{h}_{r \phi}$ would be shown to fall off as $1 / r$.

In the next step one would transform the perturbation metric to the transversal form $X_{i j}$; its components would be proportional to $\partial_{\eta}^{2} g$. Assuming that this metric is generated by weak gravitational wave sources, one would get $X_{i j} \propto \partial_{\eta}^{2} D_{i j}$, where $D_{i j}$ is the quadrupole moment of the source. Thus we can conclude that in this case the assumption that $\partial_{\eta}^{2} g_{i j}$ is discontinuous means that the fourth derivatives of the quadrupole moment are discontinuous. This can happen as a result of the action of nongravitational forces associated with violent processes. In the modern cosmological era, the nonsymmetric explosion of a supernova would constitute an example. If nonsymmetric violent events happen during the inflation or radiative era, then they would produce gravitational waves which induce permanent cosmological rotation.

Zones affected by a single pulse of radiation would appear elliptic on the cosmic microwave background radiation (CMBR) sky. Their size would depend on the time of formation - pulses created during the inflation era would be of the order (at least) of the particle horizon at the recombination era; that is, their angular sizes would be larger than (roughly) 1 degree. The rotation induced by this pulse would manifest itself through the Dopplerrelated perturbations of the spectra of the CMB radiation, which would be enclosed within the zone. Its amplitude would be strongest at the boundary of the circular 
region and weakest at its center, but the net effect would obviously depend on the orientation of the symmetry axis of the gravitational pulse and on the specific behavior of the angular velocity $\Omega$.

In the radiation era the factor $\rho_{0}^{-1} a^{-3}$ linearly increases, $\rho_{0} a^{3} \propto \eta^{-1}$. The domain of gravitational wave pulse expands and the radius of its enclosing sphere grows linearly with the cosmological time, $r \equiv \eta-\eta_{0}+r_{2}$. The linear rotational velocity of fluid particles is given by $v=\operatorname{ar} \sin \theta \Omega=C \frac{u \sin (2 \theta)}{2 r}$, where $C=-\frac{3}{2} \sqrt{\frac{5}{\pi}}$. Inserting (21), we get $\left.v \propto \eta\left(r-r_{2}+\eta_{0}\right) \partial_{\eta}^{4} g\left(r_{2}-\eta\right)\right|_{\eta_{0}} \sin (2 \theta)$. The modulus $|v|$ of linear velocity grows like $\eta$ at a fixed coordinate location $r$, but it increases like $\eta^{2}$ at points which are close to the wave front of the travelling pulse of gravitational radiation. Its amplitude can be estimated by a quantity of the order of $\frac{\left|\partial_{\eta}^{4} g\left(r_{2}-\eta\right)\right|_{\eta_{0}} \mid}{H_{0}^{2}\left(1+z_{R}\right)^{3}}$, where $H_{0}$ is the present value of the Hubble escape velocity and $z_{R}$ is the redshift at the recombination epoch.

The derivation of this bound requires two assumptions. First, we assumed a simplified evolution picture, where the Universe experiences a sudden transition from the radiation to the dust dominated epoch at the recombination time. Second, it is supposed that the gravitational radiation originates before the end of inflation era.

Combining the above estimate with the known observations of the cosmic microwave background radiation yields the bound $\left|\partial_{\eta}^{4} g\left(r_{2}-\eta\right)\right|_{\eta_{0}} \mid \leq 10^{-4} H_{0}^{2}\left(1+z_{R}\right)^{3}$; this is valid for large-scale perturbations.

\section{SUMMARY}

We have found a class of gravitational wave perturbations of radiative FLRW metrics which induces permanent rotations of the cosmological background. The smoothness of initial data is the decisive property - if initial metrics are at least $C^{2}$ everywhere, then there is no effect. If the differentiability is lower than $C^{2}$, then passing gravitational pulses enforce rotation. They might be present in the primordial (inflationary) gravitational radiation discussed in the 1980s [26] and recently reinvestigated by several researchers [27]. The observed inhomogeneities of the cosmic microwave background radiation put a bound onto discontinuities of superhorizon metric perturbations.

\section{NOTE ADDED AFTER PUBLICATION}

This and the preceding papers ([16] and [21]) show, in particular, that smooth axial gravitational waves waves do not interact with perfect fluids in FLRW spacetimes.

The related fact that axial gravitational waves would not interact with a stationary interior of barotropic stars was known at least since 1967. We read in 28]: oddparity motions are not characterized by pulsations which emit gravitational waves; rather, they are characterized by a stationary, differential rotation of the fluid inside the star and by gravitational waves which do not couple to the star at all. In 1991 S. Chandrasekhar and V. Ferrari stated that the incidence of polar gravitational waves excites fluid motions in the star while the incidence of axial gravitational waves does not (29] and papers quoted therein).

P.-M Zhang, C. Duval and P. A. Horvathy 30, have shown recently a result similar to 8, but for impulsive gravitational waves, that after the wave has passed, particles initially at rest move apart with non vanishing constant transverse velocity.

[1] A. Staruszkiewicz, Acta Phys. Pol. B 12, 327 (1981); Ann. Phys. (N.Y.) 190, 354 (1989).

[2] A. Herdegen, Lett. Math. Phys. 107, 1439 (2017).

[3] Ya. B. Zeldovitch and A. G. Polnarev, Astron. Zh. 51, 30 (1974).Sov. Astron. 18, 17 (1974).

[4] V. P. Braginsky and L. p. Grishchuk, Zh. Eksp. Teor. Fiz. 89, 744 (1985) [JETP 62, 427 (1985)].

[5] D. Christodoulou, Phys. Rev. Lett. 67, 1486 (1991).

[6] L. Blanchet and T. Damour, Phys. Rev. D 46, 4304 (1992).

[7] K. Thorne, Phys. Rev. D 45, 520 (1992).

[8] P.-M. Zhang, C. Duval, G.W. Gibbons, and P. A. Horvathy, Phys. Rev. D 96, 064013 (2017).

[9] B. P. Abbott, R. Abbott, T. D. Abbott et al., Phys. Rev. Lett. 116, 061102 (2016).

[10] P. D. Lasky, E. Thrane, Y. Levin, J. Blackman, and Y. Chen, Phys. Rev. Lett. 117, 061102 (2016).

[11] P. D. Lasky, E. Thrane, Y. Levin, J. Blackman, and Y. Chen, Phys. Rev. Lett. 118, 181103 (2017).

[12] R. Penrose in General Relativity (Papers in Honour of J. L. Synge) (Clarendon Press, Oxford, 1972), pp. 101-115.

[13] J. Luk and I. Rodnianski, Commun. Pure Appl. Math. 68, 511 (2015).

[14] T. Regge and J. A. Wheeler, Phys. Rev. 108, 1063 (1957).

[15] F. J. Zerilli, Phys. Rev. Lett. 24, 737 (1970).

[16] E. Malec and G. Wylężek, Classical Quantum Gravity 22, 3549 (2005), arXiv:gr-qc/0504110v2.

[17] E. Malec, G. Wylężek, and J. Karkowski, Gen. Relativ. Gravit. 36, 2151 (2004).

[18] G. Wylȩżek, Zasada Huygensa w kosmologii, Ph.D. thesis (Dokt. 2006/019, UJ) (2006).

[19] V. Balek and V. Polak, Gen. Relativ. Gravit. 41, 505 (2009).

[20] O. Svitek, J. Phys. Conf. Ser. 229, 012070 (2010).

[21] W. Kulczycki and E. Malec, Classical Quantum Gravity 34, 135014 (2017).

[22] S. Viaggiu, Classical Quantum Gravity 34, 035018 (2017).

[23] U. H. Gerlach and U. K. Sengupta, Phys. Rev. D 19, 2268 (1979); 22, 1300 (1980).

[24] C. Gundlach and J. M. Martin-Garcia, Phys. Rev. D 61, 
$084024(2000)$.

[25] C. Clarkson, T. Clifton, and S. February, J. Cosmol. Astropart. Phys. 06 (2009) 025, arXiv:0903.5040v2.

[26] V. A. Rubakov, M. V. Sazhin, and A. V. Veryaskin, Phys. Lett. 115B, 189 (1982); L. F. Abbott and M. B. Wise, Nucl. Phys. B244, 541 (1984); A. A. Starobinsky, Sov. Astron. Lett. 11, 133 (1985).

[27] T. Takahashi and J. Soda, Phys. Rev. Lett. 102, 231301 (2009); L. Book, M. Kamionkowski, and F. Schmidt,
Phys. Rev. Lett. 108, 211301 (2012); K.W. Masui, U.-L. Pen, and N. Turok, Phys. Rev. Lett. 118, 221301 (2017).

[28] K. S. Thorne and A. Campolattaro, Astroph. J. 149, 591(1967)

[29] S. Chandrasekhar and V. Ferrari, Royal Soc. Proc. A: Math. and Phys. Sciences 434, 449(1991).

[30] P.-M. Zhang, C. Duval and P. A. Horvathy, Memory Effect for Impulsive Gravitational Waves arXiv: $1709.02299 \mathrm{v} 2$. 